\title{
Cilostazol Improves HFD-Induced Hepatic Steatosis by Upregulating Hepatic STAMP2 Expression through AMPK
}

\author{
Yoo Jin Oh, ${ }^{1}$ Hye Young Kim, ${ }^{1}$ Mi Hwa Lee, Sung Hwan Suh, Yongmun Choi, \\ Tae-gyu Nam, Woo Young Kwon, Sang Yeob Lee, and Young Hyun Yoo \\ Departments of Anatomy and Cell Biology (Y.J.O., H.Y.K., M.H.L., W.Y.K., Y.H.Y.), Endocrinology Medicine (S.H.S.), and \\ Rheumatology (S.Y.L.), Dong-A University College of Medicine, Busan, Republic of Korea; Gyeonggi Bio Center, Gyeonggi-do \\ Business and Science Accelerator, Suwon, Republic of Korea (Y.C.); and Department of Pharmacy and Institute of \\ Pharmaceutical Science and Technology, Hanyang University, Ansan, Republic of Korea (T.-G.N.)
}

Received May 29, 2018; accepted October 5, 2018

\begin{abstract}
Nonalcoholic fatty liver disease (NAFLD) is an increasingly studied condition that can progress to end-stage liver disease. Although NAFLD was first described in 1980, a complete understanding of the mechanism and causes of this disease is still lacking. Six-transmembrane protein of prostate 2 (STAMP2) plays a role in integrating inflammatory and nutritional signals with metabolism. Our previous study suggested that STAMP2 may be a suitable target for treating NAFLD. In the current study, we performed a focused drug-screening and found that cilostazol could be a potential STAMP2 enhancer. Thus, we examined whether cilostazol alleviates NAFLD through STAMP2. The in vivo and in vitro pharmacological efficacies of cilostazol on STAMP2 expression and lipid accumulation were analyzed in
\end{abstract}

NAFLD mice induced by high-fat diet (HFD) and in HepG2 cell lines treated by oleic acid $(O A)$, respectively. Cilostazol increased the expression of STAMP2 through transcriptional regulation in vivo and in vitro. Cilostazol also dampened the STAMP2 downregulation caused by the HFD and by OA in vivo and in vitro, respectively. Cilostazol activated AMP-activated protein kinase (AMPK) in vivo and in vitro, and AMPK functions upstream of STAMP2, and reversed downregulation of STAMP2 expression through AMPK in the NAFLD model. Cilostazol ameliorates hepatic steatosis by enhancing hepatic STAMP2 expression through AMPK. Enhancing STAMP2 expression with cilostazol represents a potential therapeutic avenue for treatment of NAFLD.

\section{Introduction}

Nonalcoholic fatty liver disease (NAFLD) is one of the most common liver diseases in the world. NAFLD is characterized by fatty liver, resulting from excessive lipid accumulation in the liver. NAFLD has been known as the hepatic manifestation of metabolic syndromes such as insulin resistance (Day and James, 1998; Marchesini et al., 2003; Anstee and Goldin, 2006; Cohen et al., 2011).

Six-transmembrane protein of prostate 2 (STAMP2), which is also known as TNF-induced adipose-related protein or six-transmembrane epithelial antigen of prostate 4 (STEAP4), belongs to a family of six-transmembrane proteins called either the STAMP or STEAP family (Korkmaz et al., 2005). Although the STAMP2 protein was first identified as a prostate-specific antigen (Hubert et al., 1999), STAMP2 has

This work was financially supported by the National Research Foundation of Korea (NRF) through a grant funded by the Korean Government (MISP) [2015R1A2A1A10051603]. The authors have no conflict of interest to declare.

${ }^{1}$ Y.J.O. and H.Y.K. contributed equally to this work.

https://doi.org/10.1124/mol.118.113217. been reported to play a role in coordinating the regulation of nutrient and inflammatory responses in adipose tissue (Wellen et al., 2007; Arner et al., 2008).

STAMP2 is known to be abundantly expressed in adipose tissue, and STAMP2 expression is elevated in fed mice compared with that in fasting mice. Previous studies have shown that nutritional regulation of STAMP2 expression in adipose tissue is lost in ob/ob and diet-induced obesity models. In vitro gain-of-function studies showed that STAMP2 increases insulin sensitivity. In contrast, studies in vitro and in vivo of the loss of STAMP2 function showed that STAMP2 deficiency elevates inflammatory markers, diminishes insulin action and sensitivity, and hinders glucose uptake (Wellen et al., 2007; Abedini and Shoelson, 2007; Waki and Tontonoz, 2007).

With these previous findings as a basis, it would be useful to investigate whether STAMP2 plays a role in integrating inflammatory and nutritional signals with metabolism (Yoo et al., 2014). Previous studies demonstrated that STAMP2 is expressed in the liver (Korkmaz et al., 2005; Ramadoss et al.,

ABBREVIATIONS: Ad-STAMP2, adenoviral STAMP2; AICAR, [(2R,3S,4R,5R)-5-(4-carbamoyl-5-aminoimidazol-1-yl)-3,4-dihydroxyoxolan-2yl]methyl dihydrogen phosphate; AMPK, AMP-activated protein kinase; DMSO, dimethyl sulfoxide; FBS, fetal bovine serum; FFA, free fatty acids; HDL, high-density lipoprotein; HFD, high-fat diet; LXR $\alpha$, liver X receptor $\alpha$; NAFLD, nonalcoholic fatty liver disease; NEFA, nonesterified fatty acids; OA, oleic acid; PCR, polymerase chain reaction; p-AMPK $\alpha$, phospho-AMPK $\alpha$; RT, reverse transcription; SD, standard diet; siSTAMP2, small-interfering RNAs targeting STAMP2; SREBP-1c, sterol regulatory element-binding protein-1c; STAMP2, six-transmembrane protein of prostate 2; TC, total cholesterol. 
2010). A report demonstrating that there is notable fat accumulation and severe impairment of insulin receptor signaling in the livers of STAMP2 ${ }^{-1-}$ mice indicated that hepatic STAMP2 has a role in regulating lipid and insulin homeostasis (Wellen et al., 2007). CCAAT/enhancer-binding protein $\beta(\mathrm{C} / \mathrm{EBP} \beta)$ was shown to directly regulate the roles of STAMP2 and its novel variant in attenuating lipogenesis, gluconeogenesis, and/or inflammation elicited by free fatty acids (FFAs) or lipopolysaccharide in hepatocytes (Wang et al., 2013). A study of the hepatic role of STAMP2 demonstrated the involvement of hepatic STAMP2 in hepatitis B virus X protein-associated metabolic dysregulation (Kim et al., 2012). Our previous study illustrated that hepatic STAMP2 plays a pivotal role in preventing and improving high-fat diet (HFD)induced hepatic steatosis and insulin resistance in NAFLD (Kim et al., 2015).

In this study, we first performed a high-throughput screening to identify a STAMP2 enhancer and found that cilostazol could be a potential candidate. Thus, we examined whether cilostazol ameliorates lipid accumulation through STAMP2 in oleic acid (OA)-treated HepG2 cells and HFD-induced fatty liver NAFLD model. We observed that cilostazol improves HFD-induced hepatic steatosis by modulating STAMP2 expression through AMP-activated protein kinase (AMPK).

\section{Materials and Methods}

High-Throughput Screening. A pilot-scale screening was conducted at the Gyeonggi Bio Center (Suwon, Gyeonggi-do, Korea). HeLa cells were maintained in Dulbecco's modified Eagle's medium (DMEM) with $10 \%$ fetal bovine serum (FBS), $1 \%$ penicillin and streptomycin at $37^{\circ} \mathrm{C}$, and $5 \% \mathrm{CO}_{2}-95 \%$ air. For screening, cells were seeded into $60-\mathrm{mm}$ dished and in cultured DMEM/10\% FBS without $1 \%$ P/S. FuGENE HD transfection reagent (Roche Diagnostics, Indianapolis, IN) was used for high-throughput transfections at $1 \mu \mathrm{l}$ of FuGENE HD per $3 \mu \mathrm{g}$ of total DNA. After a 16 -hour incubation at $37^{\circ} \mathrm{C}, 4 \mu \mathrm{l}$ of the mix from each well was transferred to a 384 -well tissue culture plate and incubated for 18 hours. Luciferase was measured using the Bright-Glo Luciferase Assay (Promega, Madison, WI) and luminescence signal with an automated plate reader (PerkinElmer Envision 1; Waltham, MA). Small-molecule libraries consisting of 2000 diverse compounds were used in the screen. The compounds were selected from the DIVERSet Libraries, the Kinase Directed Library, the PremiumSet Library, and the GPCR Library (ChemBridge, San Diego, CA), and HeLa cells were treated with compounds at a $20-\mu \mathrm{M}$ final concentration for 24 hours. Oleic acid $(500 \mu \mathrm{M})$ and dimethyl sulfoxide (DMSO) were used as positive and negative controls, respectively. Primary screening identified 50 compounds with a cutoff level of a 2 -fold signal change relative to the DMSO control. To validate the 50 primary hits, confirmation screens in which each compound was tested with multiple replicates was conducted to identify 18 compounds that showed reproducible fold changes.

Materials. Cilostazol, OA, free bovine serum albumin (BSA), AICAR, Compound C, Oil Red O, and the antibodies against $\beta$-actin were purchased from MilliporeSigma (St. Louis, MO). The antibodies against sterol regulatory element-binding protein-1c (SREBP-1c) were purchased from Santa Cruz Biotechnology (Dallas, TX), the antibodies against liver $\mathrm{X}$ receptor $\alpha(\mathrm{LXR} \alpha)$ were obtained from Abcam (Cambridge, MA) and STAMP2 were obtained from Proteintech (Chicago, IL). The antibodies against AMPK $\alpha$, phospho-AMPK $\alpha$ (p-AMPK $\alpha$ ) (Thr172), HRP-conjugated goat anti-rabbit and horse anti-mouse IgG were purchased from Cell Signaling (Danvers, MA).

Animals and Treatments. Male C57BL/6 mice (22-25 g; Samtako, Inc., Osan, South Korea) were maintained on a 12-hour lightdark cycle with free access to water and food. Five-week-old mice were fed a standard diet (SD) or a HFD for 15 weeks and then randomly divided into four groups. The mice in the SD group were fed a SD and orally administered vehicle (DMSO) (SD+Veh group, $n=9$ ) or cilostazol (30 $\mathrm{mg} / \mathrm{kg}$ per day) (SD+Cilo group, $n=9$ ), whereas dyslipidemic mice were fed a HFD (HFD group, 60\% fat) (Feed Laboratory, Guri, South Korea) and received either orally administered vehicle (HFD+Veh group, $n=27$ ) or cilostazol (HFD+Cilo group, $n=21$ ). After 3, 6 and 9 weeks of treatment, the mice were anesthetized with avertin (MilliporeSigma) and sacrificed for tissue sampling after 8 hours of starvation. Blood was collected by cardiac puncture and stored at $-20^{\circ} \mathrm{C}$ in autoclaved E-tubes. Livers were isolated, immediately fixed in $10 \%$ neutral-buffered formalin solution, and stored at $4^{\circ} \mathrm{C}$ until assays were performed. All procedures were approved by the Committee on Animal Investigations at Dong-A University (DIACUC-15-12).

Cell Culture and Treatment. HepG2 cells were obtained from the American Type Culture Collection (Manassas, VA). The cells were cultured in DMEM (Gibco/Thermo Fisher Scientific, Grand Island, $\mathrm{NY})$ containing $10 \% \mathrm{FBS}$ and $1 \%(\mathrm{v} / \mathrm{v})$ penicillin-streptomycin. The cells were maintained in subconfluent conditions in a humid atmosphere of $95 \%$ air and $5 \% \mathrm{CO}_{2}$ at $37^{\circ} \mathrm{C}$. Fatty acids were conjugated with $0.1 \%$ free fatty acids (FFAs)-free BSA in DMEM. OA was added to the serum-containing cell culture medium to achieve a fatty acid concentration of $500 \mu \mathrm{M}$. HepG2 cells were treated with $500 \mu \mathrm{M}$ OA and cilostazol for 24 hours.

Histology Staining. For histopathological analyses, portions of liver were fixed ( $10 \%$ neutral-buffered formalin), dehydrated, and embedded in paraffin. Four-micrometer-thick sections were mounted on glass slides, deparaffinized in xylene, rehydrated in graded ethanol, and stained with hematoxylin and eosin. The morphology of the liver tissue was photographed using a Pannoramic MIDI-II Digital Scanner (3DHISTECH, Budapest, Hungary).

Immunohistochemical Staining. Immunohistochemistry was performed on $4-\mu \mathrm{m}$-thick liver sections. The sections were incubated with primary rabbit polyclonal STAMP2 antibody at $4^{\circ} \mathrm{C}$ overnight and then with a matching biotinylated secondary antibody for 60 minutes at $37^{\circ} \mathrm{C}$. Slides were then incubated in 3,3'-diaminobenezidine and hydrogen peroxide substrate. The negative controls did not have the primary antibody. The sections were developed with diaminobenzidine, counterstained with hematoxylin. The results were viewed using a Pannoramic MIDI-II Digital Scanner.

Oil Red O Staining. Confluent HepG2 cells were exposed to DMEM $(0.1 \%$ BSA) in the presence or absence of cilostazol with OA. After 24 hours, cells were washed in phosphate-buffered saline and fixed using $4 \%(\mathrm{w} / \mathrm{v})$ paraformaldehyde for 60 minutes at room temperature. After three washes in $60 \%$ isopropanol, the cells were stained with freshly diluted Oil Red O solution for 20 minutes. The stain was then removed, and the cells were washed four times in distilled water. To quantify intracellular lipid accumulation, Oil Red O solution was extracted using $100 \%$ isopropanol, and the absorbance was measured at $500 \mathrm{~nm}$ using a spectrophotometer.

Plasma Glucose Concentrations and Tolerance Tests for Glucose. Intraperitoneal glucose tolerance tests (IPGTT) was performed after the mice were fasted for 16 hours. Plasma glucose concentrations were measured in tail blood using a Gluco-Dr Blood Glucose Test Strip (Hasuco, Seoul, South Korea) prior to and 30, 60, 90 , and 120 minutes after intraperitoneal injection of a bolus of glucose $(1 \mathrm{mg} / \mathrm{g})$ for the IPGTT.

Analysis of Plasma Total Cholesterol Levels, Nonesterified Fatty Acid Levels, and Insulin Levels. Plasma total cholesterol (TC), and nonesterified fatty acids (NEFA) were measured with an enzymatic, colorimetric test kit (Asan Pharmaceutical Co., Seoul, Korea). Plasma insulin was detected using a mouse insulin ELISA kit (Shibayagi, Shibukawa, Gunma, Japan).

Luciferase Assay. HepG2 cells were plated in a 24-well culture plate and transfected with a reporter vector $(0.2 \mu \mathrm{g})$ together with each indicated expression plasmid using Lipofectamine 2000 (Invitrogen/ Thermo Fisher Scientific, Carlsbad, CA), according to the manufacturer's instructions. The luciferase activities were measured using the 
Dual Luciferase Reporter Assay System (Promega), according to the manufacturer's instructions. Firefly luciferase activities were standardized to Renilla activities.

RNA Isolation and Reverse Transcription-Polymerase Chain Reaction. Total RNA was prepared from cell lines or tissues using TRIzol reagent (Invitrogen), according to the manufacturer's instructions. The cDNA was synthesized from $3 \mu \mathrm{g}$ of total RNA with Moloney murine leukemia virus reverse transcriptase using a random hexamer (Cosmo Chemical Co. Ltd., Incheon, Korea) at $42^{\circ} \mathrm{C}$ for 60 minutes. The polymerase chain reaction (PCR) primers for human STAMP2 gene amplification were: 5'-CGA AAC TTC CCT CTA CCC G-3' (sense), 5'-ACA CAA ACA CCT GCC GAC TT-3' (antisense); for human $\beta$-actin gene amplification: 5 '-GAC TAC CTC ATG AAG ATC-3' (sense), 5'-GAT CCA CAT CTG CTG GAA-3' (antisense). The cDNAs were amplified by PCR under the following conditions: 30 cycles of denaturation at $95^{\circ} \mathrm{C}$ for 20 seconds, annealing at $56^{\circ} \mathrm{C}$ for 30 seconds, and extension at $72^{\circ} \mathrm{C}$ for 30 seconds in a thermal cycler.

Real-Time Polymerase Chain Reaction. A one-tenth aliquot of cDNA was subjected to PCR amplification using mouse gene-specific primers: mouse Stamp2, 5'-GGT TGT CTG CAT TTT CGG AAC-3' (sense), 5'-GGT TTC GAC TCC CAA AAA CGA-3' (antisense); mouse Gapdh, 5'-AGG TCG GTG TGA ACG GAT TTG-3' (sense), 5'-GGG GTC GTT GAT GGC AAC A-3' (antisense). Real-time PCR was performed using SYBR Green PCR Master Mix (Applied Biosystems, Foster City, CA) with an ABI 7500 instrument (Applied Biosystems).

Western Blot Analysis. Cells were washed with phosphatebuffered saline, resuspended in ice-cold lysis buffer and incubated at $4^{\circ} \mathrm{C}$ for 20 minutes. Lysates were centrifuged at $13,000 \mathrm{rpm}$ for 20 minutes at $4^{\circ} \mathrm{C}$. Twenty microgram of proteins was loaded onto 7.5\%-15\% SDS-PAGE. The proteins were transferred onto nitrocellulose membrane (Amersham Pharmacia Biotech, Piscataway, NJ) and reacted with each antibody. Western blot analysis with antibodies was performed with SuperSignal West Pico (Thermo Fisher Scientific, Waltham, MA) enhanced chemiluminescence substrate and detected using the LAS-3000 Plus (Fuji Photo Film, Tokyo, Japan). Quantification and normalization to actin control bands using Image $\mathrm{J}$ version $1.48 \mathrm{q}$.

Nile Red Staining. Cells were fixed with $4 \%$ paraformaldehyde for 60 minutes and stained with Nile Red $(1 \mu \mathrm{g} / \mathrm{ml})$ in the dark for 10 minutes at room temperature. The red and green fluorescence signals in the cells were imaged using a Zeiss LSM 800 laser-scanning confocal microscope (Göttingen, Germany).

RNA Interference and Transfection. For the small-interfering RNA-mediated downregulation of STAMP2, small-interfering RNAs targeting STAMP2 (siSTAMP2), and negative control small conditional RNA were purchased from MilliporeSigma. HepG2 cells were transfected with either siRNA specific for STAMP2 or a negative control small conditional RNA using Lipofectamine RNAi MAX (Invitrogen) according to the manufacturer's instructions.

Adenoviral STAMP2 Infection. Recombinant adenoviral STAMP2 (Ad-STAMP2) was prepared as described previously (Kim et al., 2015). HepG2 cells were infected with recombinant Ad-STAMP2 at multiplicity of infection (MOIs) of 100.

Statistical Analysis. The results are expressed as the means \pm S.D. from three experiments. Statistical significance between two groups was determined by unpaired two-tailed Student's $t$ test. For comparison among multiple groups, at least three independent experiments in triplicate were conducted. Shapiro-Wilk test was conducted to check normality of data and Levene's test verified homogeneity of variances before one-way analysis of variance. Analysis of variance followed by Scheffe's test was used for the analysis of differences within each treated conditions. $P<0.05$ indicated statistical significance.

\section{Results}

High-Throughput Screening. A pilot-scale screening was conducted at the Gyeonggi Bio Center. Renilla luciferase activity driven by STAMP2 expression was measured.
Primary screening found 41 upregulating compounds and 9 downregulating compounds with 2-fold or greater signal changes in the STAMP2 expression level relative to the DMSO control. To test the reproducibility and specificity of the 50 primary hits, we conducted a confirmation screening in which each compound was tested with multiple replicates. As a result, 18 compounds were validated as STAMP2 modulators: 13 upregulators (Fig. 1) and 5 downregulators. Among the 13 upregulators identified through the confirmation screening and additional assays, potential Michael acceptors (conjugated nitriles), metal chelators (hydroxyquinolines), and intercalators (polycyclic aromatics) were excluded to focus on a few chemical scaffolds of interest. Of these scaffolds, the 3,4-dihydroquinolin2-one scaffold was commonly encountered in three hits. This scaffold is interesting and promising because it features a simple and flexible platform for derivatization from a drug discovery point of view. All three compounds containing 3,4dihydroquinolin-2-one show acceptable cLogP values ranging from 3.24 to 3.96 .

Cilostazol Increases Hepatic STAMP2 Expression In Vivo and In Vitro. We wanted to use more drug-like derivatives for further characterization. Thus, we performed an extensive structure search. We finally found that among marketed drugs, cilostazol has the same chemical scaffold. Because cilostazol has been widely used without significant complications in the clinical field, we chose this drug for further study. Immunohistochemical analysis (Fig. 2A), real time PCR (Fig. 2B), and Western blot assay (Fig. 2C) demonstrated that cilostazol (30 $\mathrm{mg} / \mathrm{kg}$ per day) increased the expression of STAMP2 in the liver of C57BL/6 mice. Relative luciferase assay showed that cilostazol enhanced the promoter activity of STAMP2 in HepG2 cells in a dose- and time-dependent manners (Fig. 2, D and E). RT-PCR and Western blot analysis showed that cilostazol at 10-100 $\mu \mathrm{M}$ enhanced the expression of STAMP2 mRNA and protein in HepG2 cells, respectively (Fig. 2, F and G). The concentrations used for in vitro and in vivo studies were comparable to clinical levels (Ota et al., 2008).

Cilostazol Attenuates HFD-Induced NAFLD in Mice. To investigate the effects of cilostazol in NAFLD mice, male C57BL/6 mice were randomly divided into four groups: 1) SD+ Veh, 2) SD+Cilo, 3) HFD+Veh, and 4) HFD+Cilo (Fig. 3A). We examined whether cilostazol modulates insulin sensitivity and glucose metabolism in HFD-induced NAFLD mice. Cilostazol treatment of 9 weeks reversed the increase in blood glucose levels and plasma insulin levels induced by the HFD. Furthermore, cilostazol treatment significantly lowered blood glucose levels in glucose tolerance test (Fig. 3B). Observation of the bodies and the livers of the mice showed that cilostazol treatment attenuated HFD-induced accumulation of lipids in the liver and the abdominal viscera. Cilostazol treatment of 9 weeks significantly reversed the HFD-induced increase in the ratio of liver to body weight (Fig. 3C). Liver histology by hematoxylin and eosin staining showed that cilostazol treatment of 9 weeks markedly attenuated HFD-induced aberrant lipid accumulation (Fig. 3C). Although plasma TC and NEFA levels were increased in mice fed a HFD compared with those in mice fed a SD, cilostazol treatment of 9 weeks reversed the increase in plasma TC and NEFA levels induced by the HFD (Fig. 3D). These phenotypes were not observed at 3 and 6 weeks following cilostazol treatment (data not shown). HFD-induced increase of the expression level of SREBP-1c 
A
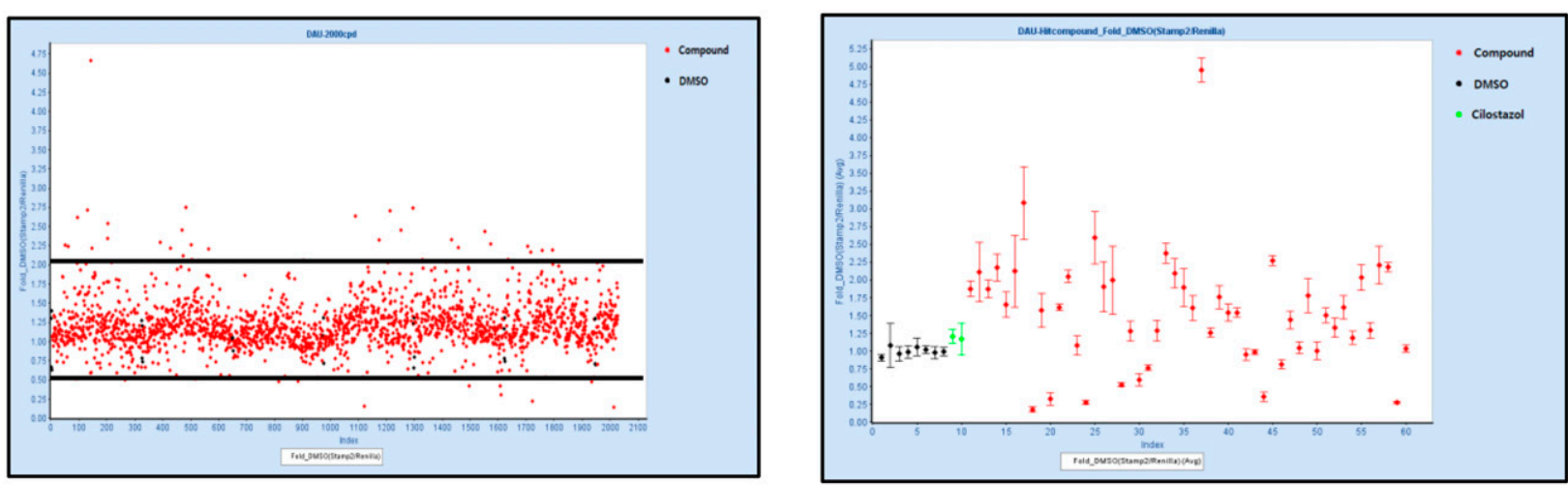

B

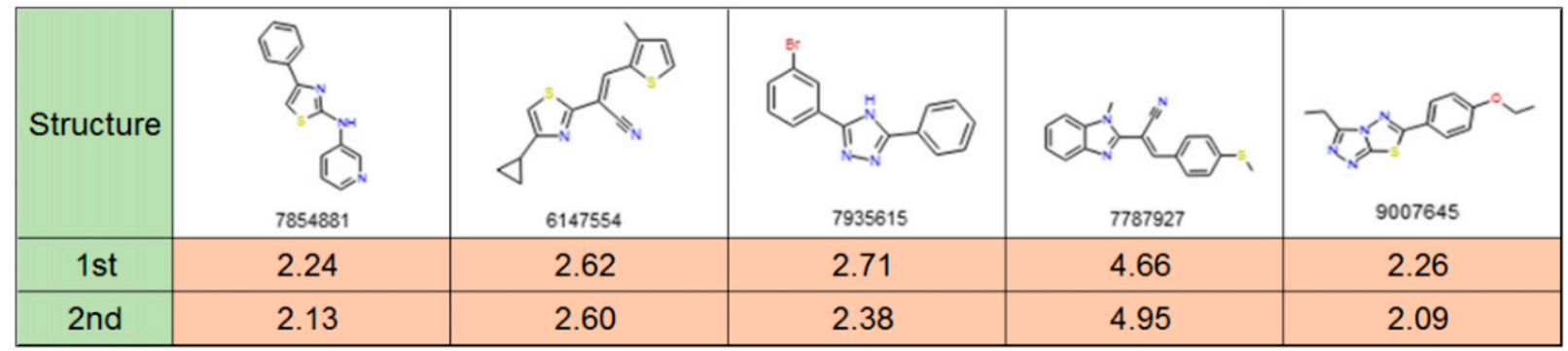

\begin{tabular}{|c|c|c|c|c|c|}
\hline Structure & & \\
\hline 1st & 2.23 & 2.06 & 2.44 & 2.34 & 2.29 \\
\hline 2nd & 2.04 & 2.18 & 2.18 & 2.27 & 2.21 \\
\hline
\end{tabular}

\begin{tabular}{|c|c|c|c|}
\hline & \\
\hline Structure & \\
\hline 1st & 2.22 & 2.45 & 2.12 \\
\hline 2nd & 2.11 & 3.09 & 2.05 \\
\hline
\end{tabular}

Fig. 1. High throughput screening. (A) First screening (Singlet) (left). Confirmation screening (Triplicate) (right). To quantify the selectivity of compounds in the former class, as well as uncover compounds in the nonselective class that might display selectivity at lower doses, all 50 compounds were entered into a secondary screen using a drug concentration range. (B) Confirmation screening identified 13 upregulator compounds with 2 -fold or greater selectivity.

and LXR $\alpha$, which are key lipogenic factors, was significantly reversed by cilostazol treatment (Fig. 3E). These in vivo and in vitro assays indicate that cilostazol improves hepatic steatosis and insulin resistance in HFD-induced NAFLD in mice.

Cilostazol Ameliorates Lipid Accumulation through Hepatic STAMP2. Immunohistochemical analysis and Western blot analysis demonstrated that the expression of hepatic STAMP2 was markedly reduced in liver tissue obtained from mice fed a HFD compared with mice fed a SD. Importantly, cilostazol reversed the reduced expression of hepatic STAMP2 in mice fed a HFD (Fig. 4A). High levels of plasma FFAs are implicated in the pathogenesis of NAFLD, because FFAs can accumulate in hepatocytes to form lipid droplets. This condition was mimicked in vitro by treating HepG2 cells with $500 \mu \mathrm{M}$ OA. The HepG2 cells were incubated with $500 \mu \mathrm{M}$ OA with or without cilostazol for 24 hours. Oil Red $\mathrm{O}$ staining was performed to demonstrate whether cilostazol influenced lipid accumulation in the HepG2 cells treated with OA. Lipid accumulation was increased in the HepG2 cells incubated with $500 \mu \mathrm{M}$ OA alone. Noticeably, cilostazol significantly reversed the OA-induced lipid accumulation (Fig. 4B). Western blot analysis showed that the expression levels of SREBP-1c and $\mathrm{LXR} \alpha$ proteins were 
A
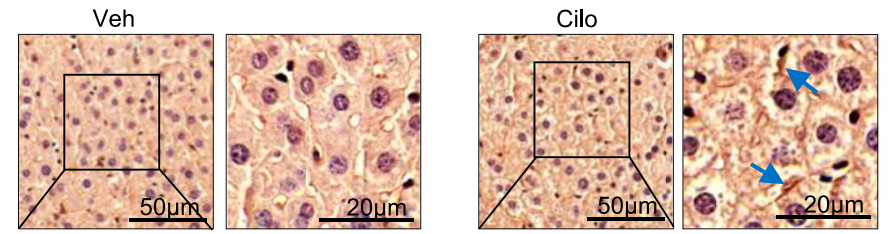

B

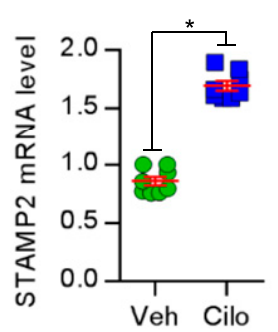

C
D

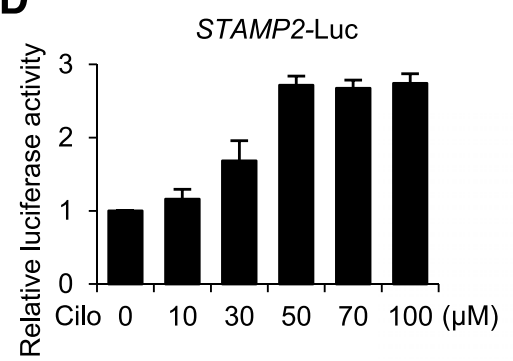

$\mathbf{E}$

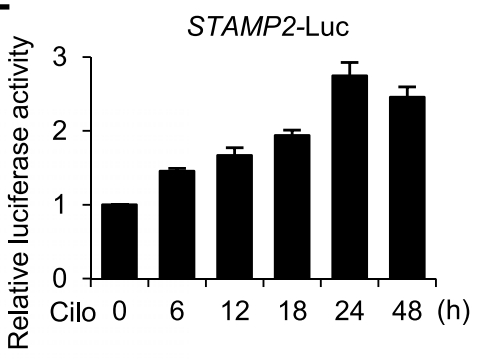

Fig. 2. Cilostazol increases hepatic STAMP2 through transcriptional regulation in vivo and in vitro. (A) Immunohistochemical staining, (B) real-time PCR, and (C) Western blot assay and quantification showing the effect of cilostazol treatment of 9 weeks on the expression of STAMP2 in liver tissue obtained from C57BL/6 mice. $n=9$ per group. Veh, vehicle. ${ }^{*} P<0.05$. (D) Luciferase assays showing the effects of $0-100 \mu \mathrm{M}$ cilostazol (Cilo) on STAMP2 promoter activity in HepG2 cells. (E) Time-sequenced luciferase assays showing the effects of $50 \mu \mathrm{M}$ cilostazol on STAMP2 promoter activity in HepG2 cells. ( $\mathrm{F}$ and $\mathrm{G}$ ) The effect of cilostazol on the expression of STAMP2 mRNA and protein in HepG2 cells. (F) RT- PCR. (G) Western blot analysis.

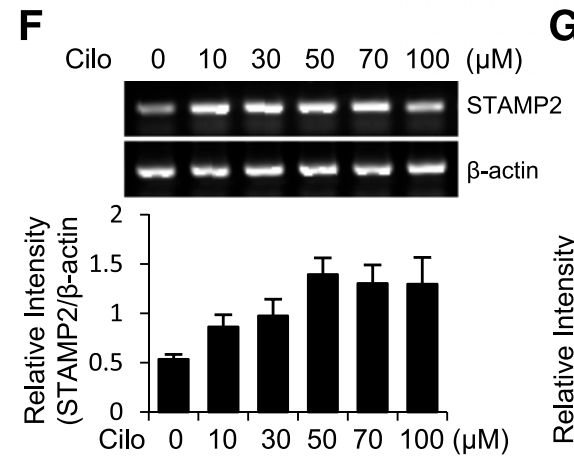

G
Cilo $\quad 0 \quad 10 \quad 30 \quad 50 \quad 70 \quad 100(\mu \mathrm{M})$

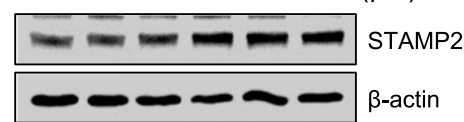

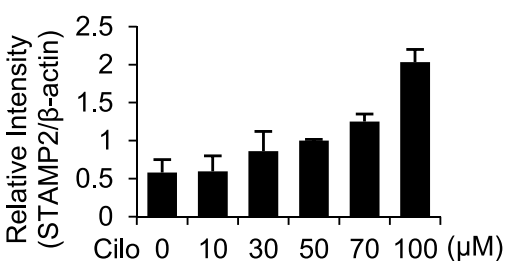

increased by OA treatment, which was significantly reversed by cilostazol treatment in HepG2 cells (Fig. 4B). Cilostazol also reversed the reduction in STAMP2 expression caused by $\mathrm{OA}$, as shown by the relative luciferase assays and Western blot analysis (Fig. 4C). To prove that cilostazol ameliorates lipid accumulation through STAMP2, we performed a STAMP2 knockdown assay in vitro. A Western blot analysis showed that STAMP2 was efficiently depleted by silencing of STAMP2 (Fig. 4D). Notably, siSTAMP2 prevented the amelioration of OA-induced lipid accumulation caused by cilostazol (Fig. 4E). We examined whether siSTAMP2 modulates the effect of cilostazol on the expression of SREBP-1c and LXR $\alpha$. siSTAMP2 reversed the attenuation of their expression by cilostazol (Fig. 4E). These findings suggest that improvement of hepatic steatosis by cilostazol may be associated with the restoration of STAMP2 expression.

AMPK Is Involved in the Amelioration of Lipid Accumulation by Cilostazol. AMPK plays a pivotal role in the functional network of hepatic steatosis (Kohjima et al., 2008). Thus, we next examined whether cilostazol ameliorates lipid accumulation through AMPK. We observed that a HFD significantly reduced the expression level of p-AMPK $\alpha$ compared with the experimental control (SD group). We further observed that cilostazol increased the expression level of p-AMPK $\alpha$ not only in mice fed a SD but also in mice fed a HFD (Fig. 5A). Additionally, cilostazol increased the expression level of p-AMPK $\alpha$ in OA-induced HepG2 cells (Fig. 5B). To prove that the efficacy of cilostazol is mediated through AMPK, we examined the effects of an AMPK activator AICAR or an AMPK inhibitor Compound $\mathrm{C}$ on cilostazol treatment. Whereas the cilostazol-induced attenuation of lipid accumulation and the expression level of SREBP-1c and LXR $\alpha$ proteins was reversed by Compound $\mathrm{C}$, the effects were augmented by AICAR (Fig. 5, C and D). These data indicate that AMPK is involved in the amelioration of lipid accumulation by cilostazol.

AMPK Functions Upstream of STAMP2. We next examined whether AMPK activation modulates hepatic STAMP2 expression. Relative luciferase assay (Fig. 6A) and Western blot analysis (Fig. 6B) showed that the expression of hepatic STAMP2 was significantly increased by AICAR but was decreased by Compound $\mathrm{C}$ (Fig. 6, A and B). Relative 
A
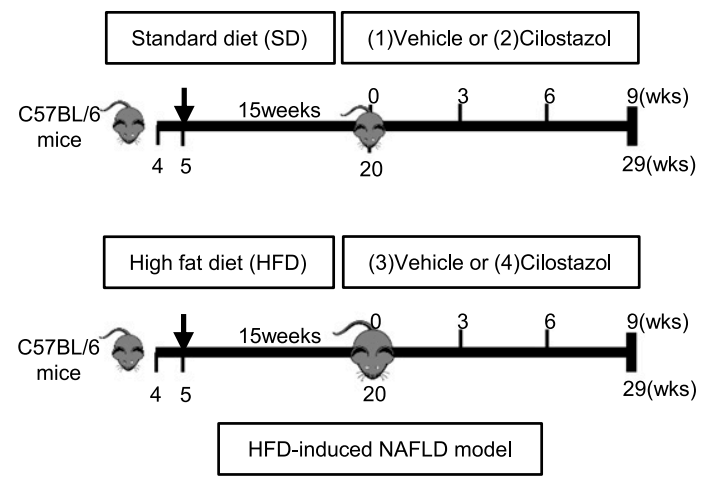

C

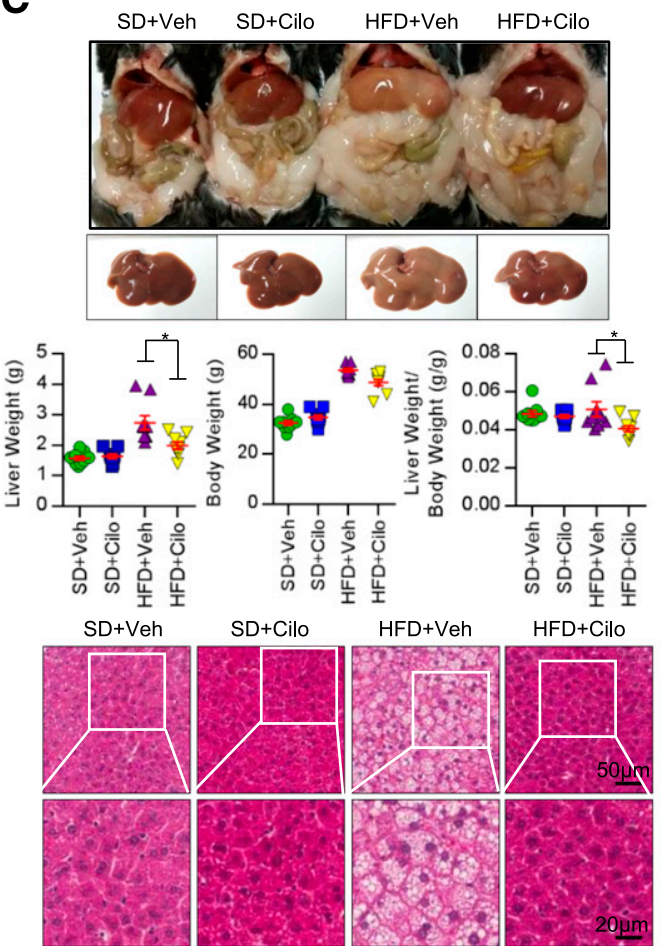

B
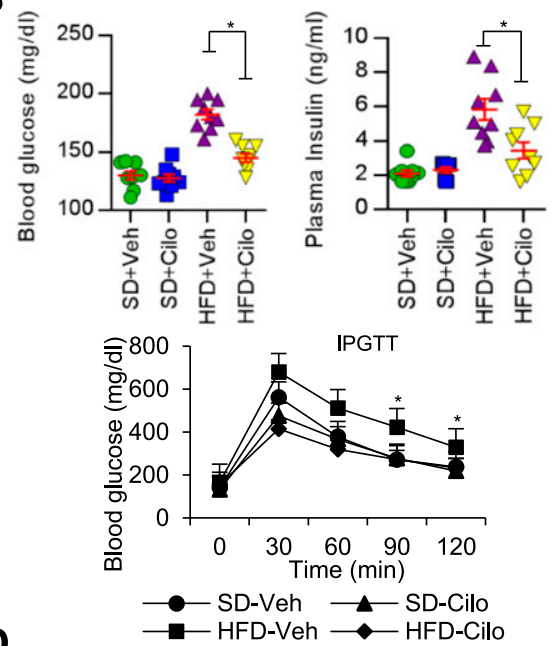

D

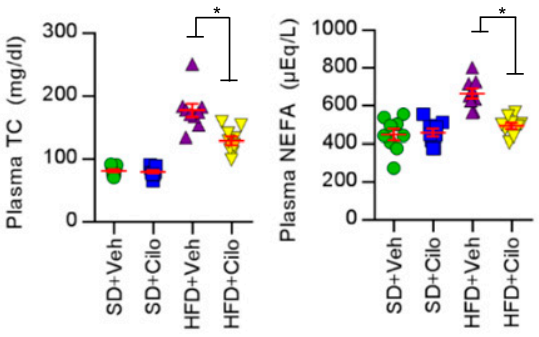

E

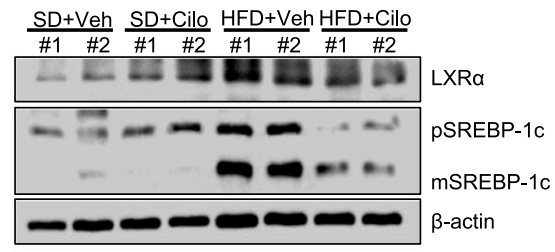

- LXRa ISREBP-1c

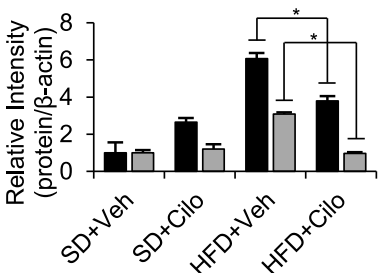

Fig. 3. Cilostazol attenuates HFD-induced NAFLD in mice. (A) Experimental design. (B) Blood glucose and plasma insulin levels, and IPGTT. Assays were performed on SD- and HFD-fed mice with or without cilostazol for 9 weeks. $n=9$ per group. (C) Representative livers obtained 9 weeks after treatment with cilostazol or vehicle. $n=9$ per group. Liver and body weights as well as the ratio of liver to body weight are illustrated (upper panel). Hematoxylin and eosin staining performed for the assessment of liver histology (lower panel). (D) Measurement of plasma TC and plasma NEFA levels. $n=9$ per group. (E) Western blot analysis and quantification of the hepatic expression of LXR $\alpha$ and SREBP-1c. $n=9$ per group. ${ }^{*} P<0.05$.

luciferase assay showed that AICAR and Compound C significantly reversed oleic acid-induced downregulation and cilostazol-induced upregulation of STAMP2, respectively (Fig. $6 \mathrm{C})$. Western blot assay also showed that the ability of cilostazol to reverse the OA-induced decrease in STAMP2 expression was inhibited by Compound $\mathrm{C}$ but was augmented by AICAR (Fig. 6D). We observed that siSTAMP2 had no effect on the AICAR-induced increase of p-AMPK $\alpha$ (Fig. 6E). We further observed that cilostazol increased p-AMPK $\alpha$ levels regardless of STAMP2 knockdown in OA-treated HepG2 cells (Fig. 6F). Noticeably, siSTAMP2 reversed the amelioration of OA-induced lipid accumulation by AICAR (Fig. $6 \mathrm{G})$. We observed that Ad-STAMP2 had no effect on the
AICAR-induced increase of p-AMPK $\alpha$ (Fig. $6 \mathrm{H}$ ). We further observed that cilostazol increased p-AMPK $\alpha$ levels regardless of STAMP2 overexpression in OA-treated HepG2 cells (Fig. 6I). Noticeably, Ad-STAMP2 reversed the augmentation of OA-induced lipid accumulation by Compound C (Fig. 6J). These data, in conjunction with Fig. 5B, show that AMPK functions upstream of STAMP2 for the amelioration of lipid accumulation by cilostazol.

\section{Discussion}

NAFLD is a spectrum of disorders ranging from isolated hepatic steatosis to nonalcoholic steatohepatitis, which is the 
A

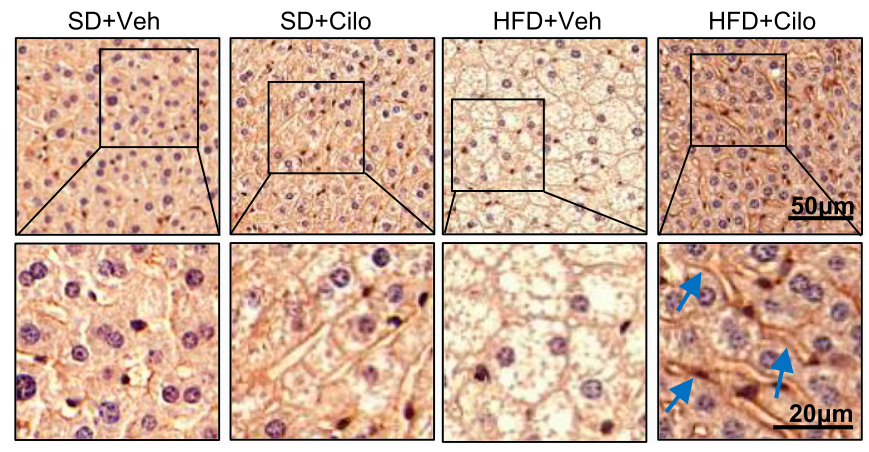

B

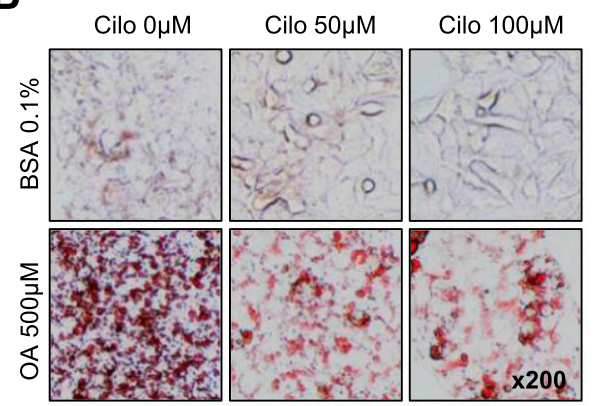

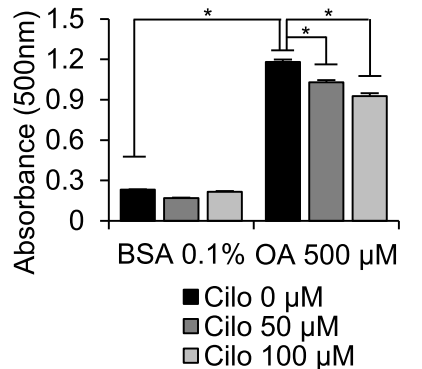

$\square$ Cilo $100 \mu \mathrm{M}$

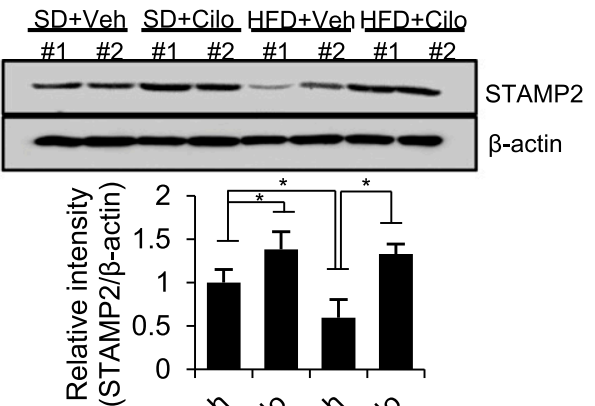

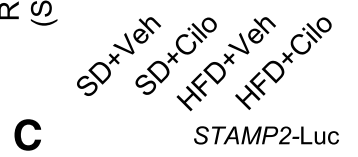
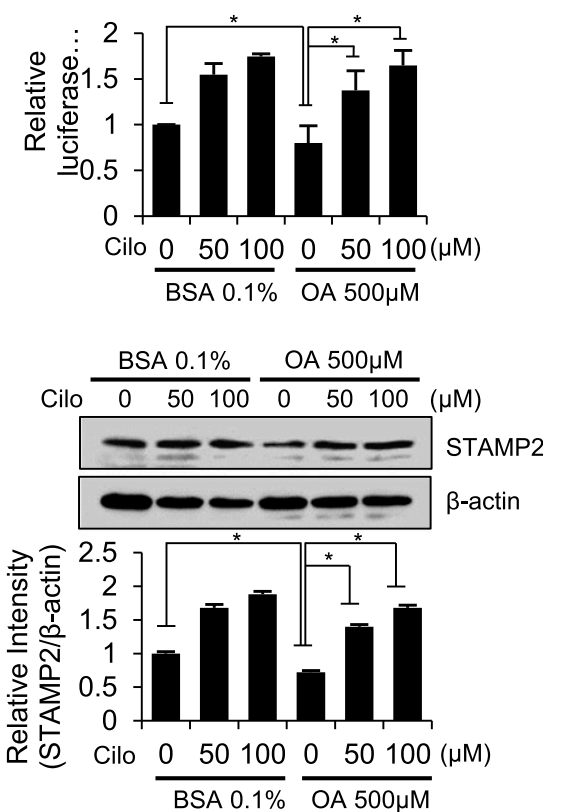

D

E
- LXRa $\square$ SREBP-1C
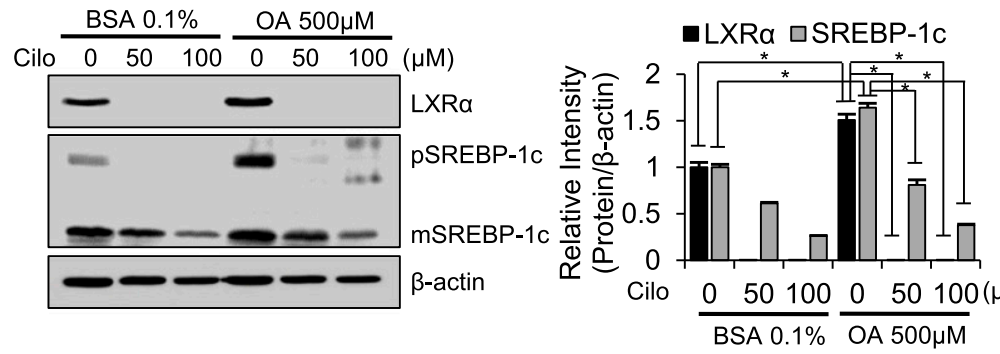

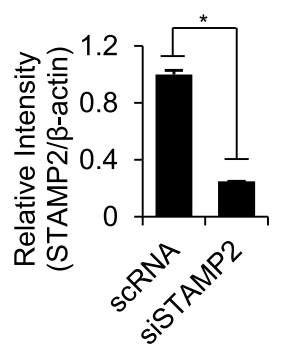

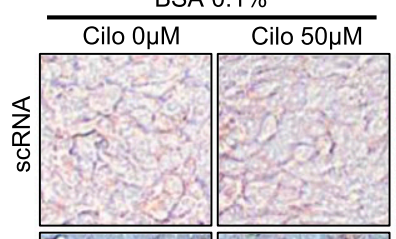

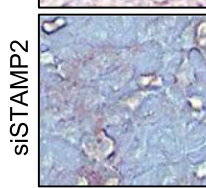

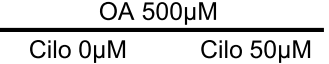
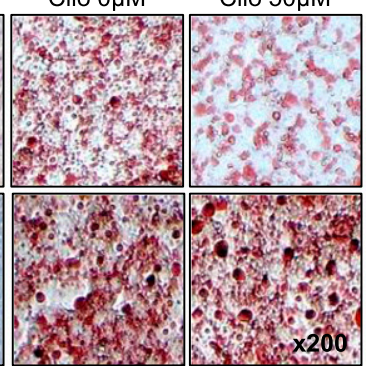

- Cilo 0

口Cilo 50

$\square$ Cilo 0+OA 口Cilo 50+OA

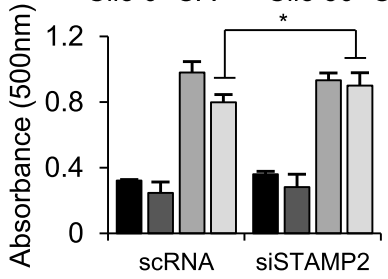

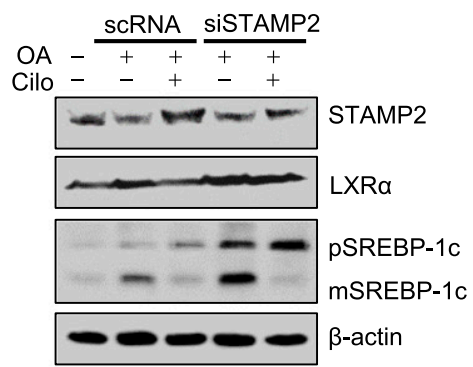

- LXRa $\quad$ SREBP-1C

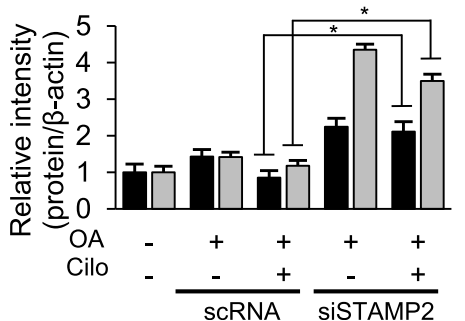

Fig. 4. Cilostazol ameliorates lipid accumulation through hepatic STAMP2. (A) Immunohistochemical staining and Western blot assay and quantification. The effect of cilostazol on the expression of STAMP2 in the liver tissue obtained from C57BL/6 mice. $n=9$ per group. (B) Oil Red O staining and Western blot analysis, and their quantification showing the effect of cilostazol treatment on the OA-induced lipid accumulation and lipogenic-related protein expression level in HepG2 cells. (C) Luciferase assay and Western blot analysis and their quantification. The effect of 
A

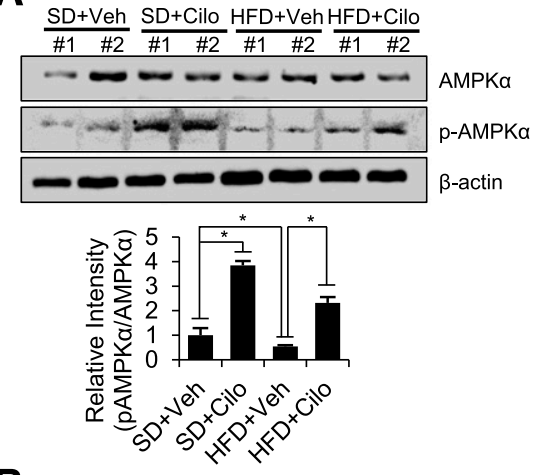

B

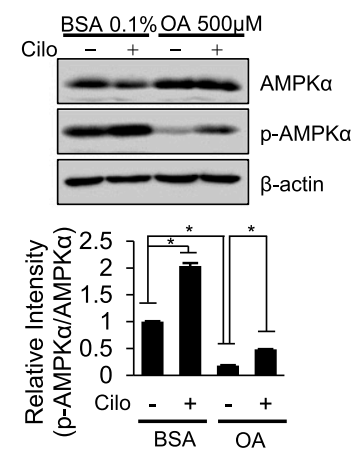

C

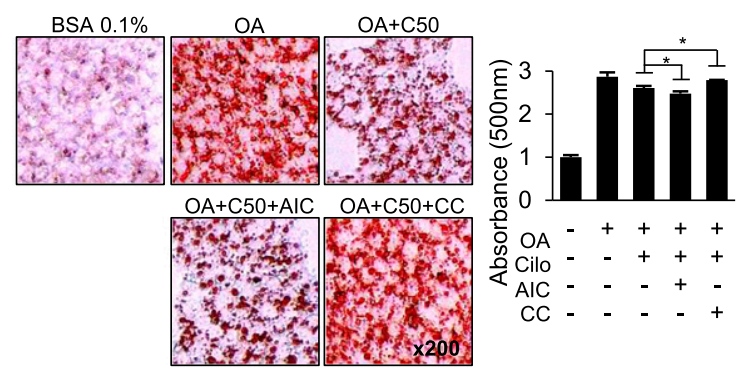

D

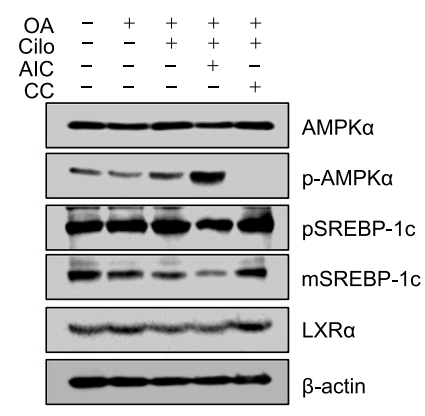

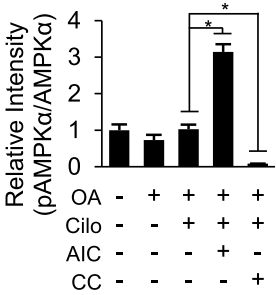

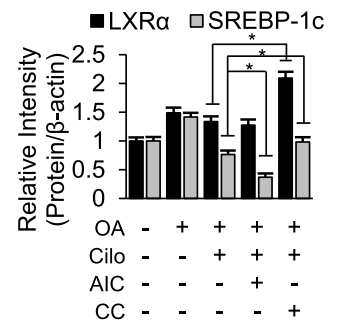

Fig. 5. AMPK is involved in the amelioration of lipid accumulation by cilostazol. (A and B) Western blot analysis and quantification showing the effect of cilostazol on the expression of p-AMPK $\alpha$. The liver of C57BL/6 mice. $n=9$ per group (A). HepG2 cells (B). (C and D) The effect of AICAR (AIC) or Compound C (CC) on the amelioration by cilostazol of lipid accumulation. Oil Red O staining and quantification (C) and Western blot analysis and quantification of the expression of LXR $\alpha$ and SREBP-1c (D). $* P<0.05$.

more aggressive form of fatty liver disease that may progress to cirrhosis and cirrhosis-related complications, including hepatocellular carcinoma (Bugianesi et al., 2002). NAFLD is strongly associated with the risk of more severe conditions such as cardiovascular disease and atherosclerosis (Targher 2007). Overweight, insulin resistance, a sedentary life-style, and an altered dietary pattern, as well as genetic factors and disturbances in intestinal barrier function are key risk factors for NAFLD. Despite intensive studies of the pathogenesis of NAFLD, the initiation of the disease and the detailed molecular mechanisms involved in its progression are not entirely understood (Kanuri and Bergheim, 2013).

The primary treatment of NAFLD is weight reduction. However, because it is hard to maintain weight loss, several pharmacotherapies have been considered for NAFLD patients. New classes of medications have been developed in recent years on the basis of an understanding of disease pathogenesis and a drug repurposing strategy that has identified several currently available agents (Rotman and Sanyal, 2017). Some new agents that activate or inhibit nuclear receptor signaling, such as peroxisome proliferatoractivated receptor $\beta / \delta(\operatorname{PPAR} \beta / \delta), \operatorname{PPAR} \gamma$, farnesoid $\mathrm{X}$ receptor, and LXR, have shown clinical evidence of therapeutic benefit (Tanaka et al., 2017). However, most drugs under evaluation have not resulted in the solution of the complex nature of NAFLD (Wong et al., 2016), and no existing agent has been specifically approved for treating NAFLD and nonalcoholic steatohepatitis (Cusi, 2016). Thus, development or identification of agents for treating this disease is an urgent challenging task.

STAMP2 plays a role in integrating inflammatory and nutritional signals with metabolism. An elaborate study demonstrated that STAMP2 expression is responsive to both nutritional and inflammatory signals. The study further showed that STAMP2 deficiency induces the accumulation of extra body fat and systemic insulin resistance. (Wellen et al., 2007). STAMP2 plays a critical function in metabolic and inflammatory pathways by acting in adipocytes and macrophages, which are required for normal metabolic homeostasis (ten Freyhaus et al., 2012). Our previous study revealed that deficiency of hepatic STAMP2 significantly accelerates hepatic steatosis and insulin resistance in HFD-induced NAFLD mice, indicating that STAMP2 may represent a suitable target for interventions in NAFLD (Kim et al., 2015).

Cilostazol is an antiplatelet drug that inhibits both primary and secondary platelet aggregation in response to ADP, 
A
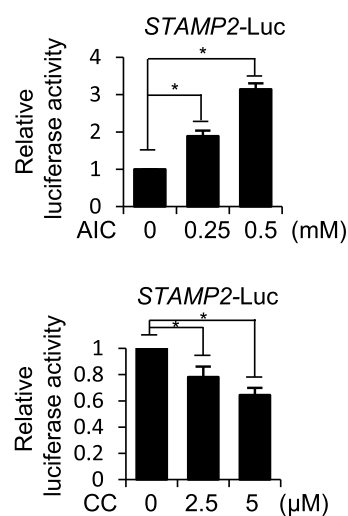

E
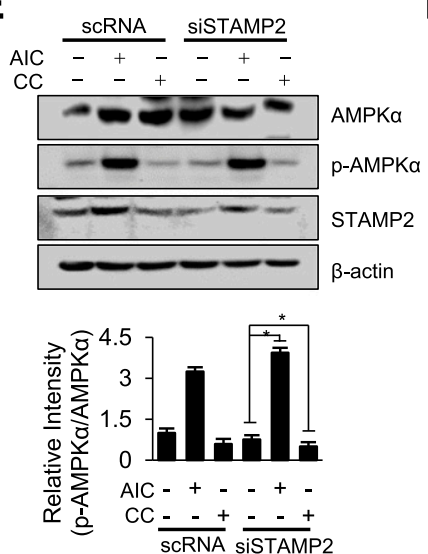

H
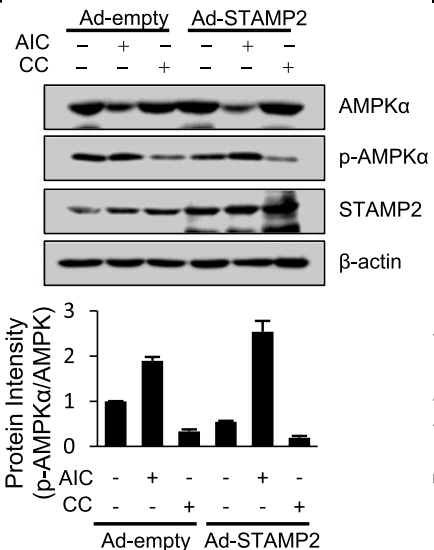

B
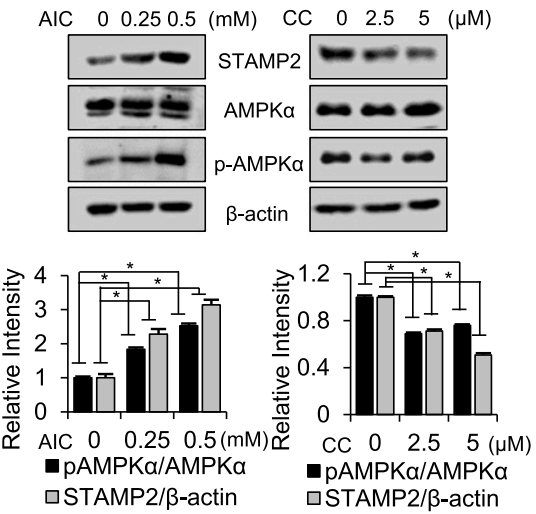

F

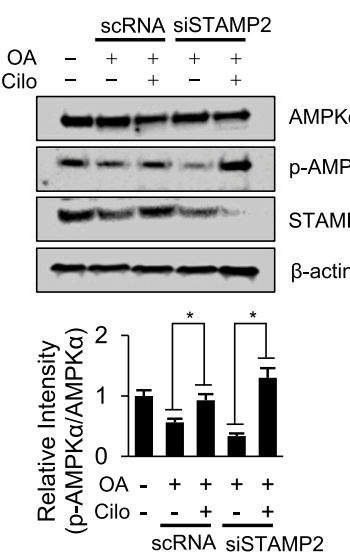

I
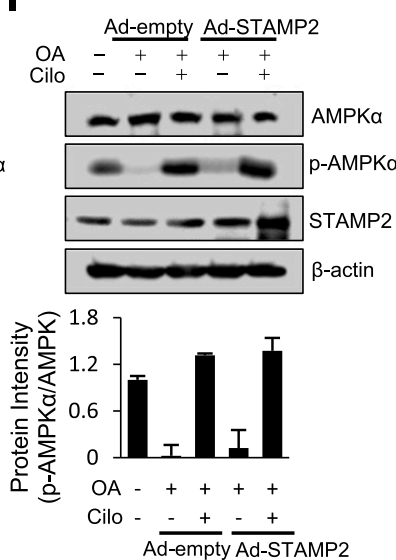

G
C

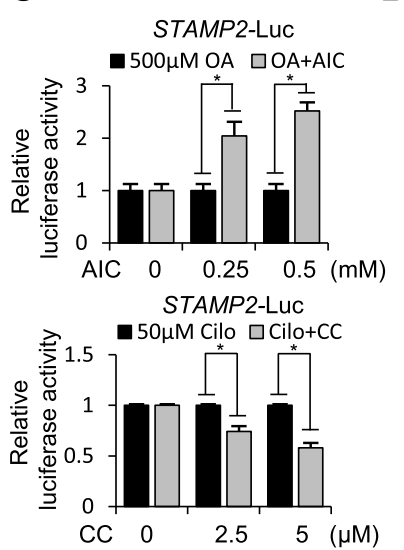

D
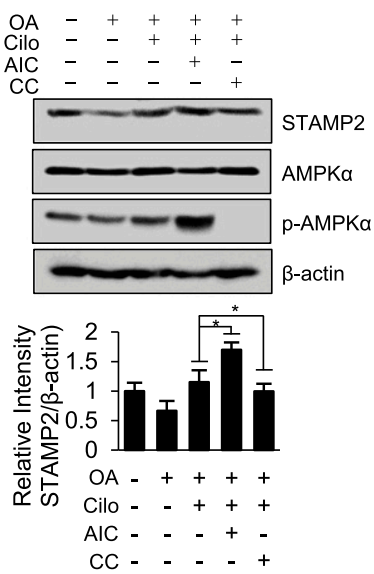

Fig. 6. AMPK functions upstream of STAMP2. (A and B) The effect of AICAR or Compound C on the expression of STAMP2. (A) Relative luciferase assay. (B) Western blot analysis. (C) Relative luciferase assay showing that AICAR reversed oleic acid-induced downregulation and that Compound $\mathrm{C}$ reversed cilostazol-induced upregulation of STAMP2. (D) Western blot analysis and quantification showing that the ability of cilostazol to reverse the OA-induced decrease in STAMP2 expression was inhibited by Compound C but was augmented by AICAR. (E and F) Western blot analysis and quantification of the expression of p-AMPK $\alpha$ in STAMP2-depleted cells (siSTAMP2). The effect of AICAR or Compound C (E). The effect of cilostazol (F). (G) Oil Red O staining and quantification showing the effect of AICAR in STAMP2-depleted cells. (H and I) Western blot analysis and quantification showing the effect of Ad-STAMP2 on the expression of p-AMPK $\alpha$ in AICAR- or Compound C-treated (H) and cilostazoltreated (I) cells. (J) Oil Red O staining and quantification showing the effect of Ad-STAMP2 on Compound C-treated cells. *P $<0.05$.

collagen, epinephrine, and arachidonic acid (Kimura et al., 1985). The antiplatelet and vasodilator properties of cilostazol are attributed to its ability to elevate intracellular levels of cyclic AMP (cAMP) via inhibition of cAMP phosphodiesterase (Okuda et al., 1993). Cilostazol is now being used for the treatment of intermittent claudication (Beebe et al., 1999).
Furthermore, several more recent meta-analyses revealed that cilostazol, compared with aspirin, not only has similar or even slightly better therapeutic effects in stroke prevention but also caused a significant reduction in hemorrhagic stroke (Dinicolantonio et al., 2013). Previous studies have reported that cilostazol contributes to the amelioration of dyslipidemia 
(Elam et al., 1998). In addition, early studies performed in Japan and the United States demonstrated a beneficial effect of cilostazol on lipoprotein metabolism characterized by an increase in high-density lipoprotein-cholesterol (HDL-C) and a reduction of plasma triglyceride (TG) levels (Elam et al., 1998; Tani et al., 2000; Ikewaki et al., 2002). Cilostazol seems to increase HDL-C levels, which contributes to the amelioration of dyslipidemia. However, the mechanism underlying the pharmacological efficacy of cilostazol has not been fully elucidated in the liver.

Previous studies have shown that cilostazol has beneficial effects on glucose metabolism both in vitro and in vivo (Park et al., 2008, 2009; Kim et al., 2014). Adipose tissue remodeling and inflammation are key metabolic events associated with obesity-related insulin resistance (Osborn and Olefsky, 2012). Cilostazol attenuates $\mathrm{TNF} \alpha$-induced chronic inflammation in adipose tissue through suppression of $\mathrm{TNF} \alpha$ production from macrophages, which leads to amelioration of systemic insulin resistance in obese diabetic mice (Wada et al., 2013).

Several previous reports have shown that cilostazol ameliorates lipid imbalances in NAFLD. Cilostazol appeared to exert beneficial effects against NAFLD (Fujita et al., 2008). Recently, Jung et al. (2014) demonstrated that cilostazol inhibits insulin-induced hepatic SREBP-1c expression via the inhibition of LXR and Sp1 activity, and suggested that cilostazol negatively regulates hepatic lipogenesis through the inhibition of SREBP-1c transcription. Another study showed that cilostazol ameliorated hepatic steatosis and increased ATP-binding cassette transporter A1 expression in the hepatocytes (Jeon et al., 2015). However, the precise mechanism underlying the amelioration by cilostazol of lipid imbalance in NAFLD has not been clarified. We, in the present study, demonstrated that cilostazol improves lipid accumulation in NAFLD models through STAMP2. We observed that cilostazol treatment augments hepatic STAMP2 expression, which led to hepatic steatosis amelioration. In addition, we observed that hepatic STAMP2 silencing prevented cilostazol from reducing lipid accumulation in HepG2 cells.

AMPK phosphorylates key metabolic enzymes and transcriptional regulators such as fatty acid synthase, SREBP1, SREBP2, and acetyl-CoA carboxylase (ACC) that are linked to controlling lipid biosynthesis (Day et al., 2017). Previous studies have shown that cilostazol activates AMPK in vascular smooth muscle and endothelial cells (Suzuki et al., 2008; Aoki et al., 2010). In the present study, we demonstrated that cilostazol activated AMPK and ameliorated lipid imbalances in NAFLD. In addition, we demonstrated that cilostazol increased the expression of hepatic STAMP2 through AMPK (Fig. 7). Irrespective of our data, our study lacked in vivo lossof-function assessment. We had previously demonstrated that liver-specific deletion of STAMP2 accelerates hepatic steatosis in HFD-induced NAFLD mice (Kim et al., 2015). However, we did not adopt liver-specific deletion of STAMP2 for this study because the effect of cilostazol was evident after 9 weeks of cilostazol treatment, whereas the effect of liver-specific deletion would last for 2 weeks at most. Thus, to prove that cilostazol ameliorates lipid accumulation through STAMP2, further research using STAMP2 knockout mice is required.

In conclusion, cilostazol increases hepatic STAMP2 expression and ameliorates hepatic steatosis not only in the liver of HFD-induced NAFLD mouse model but in HepG2 cells treated with OA. In addition, cilostazol improves HFD-induced

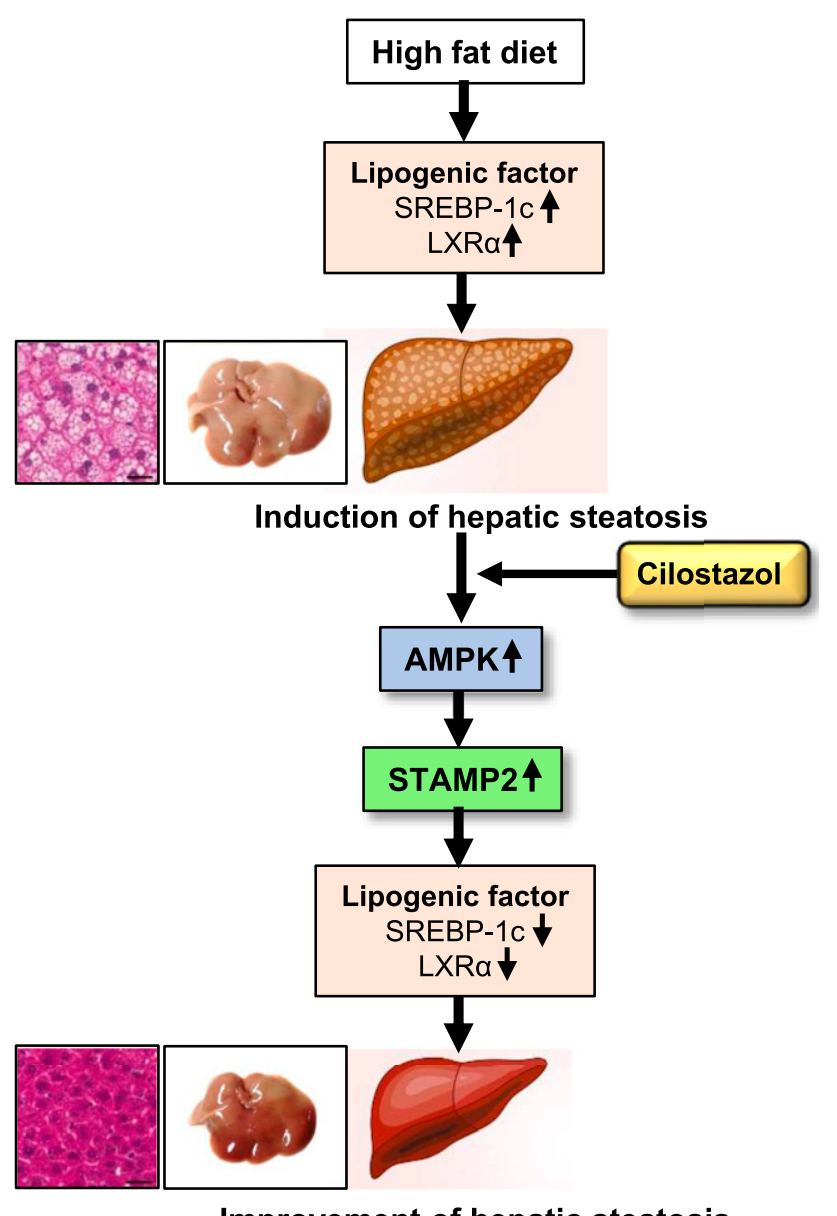

Improvement of hepatic steatosis

Fig. 7. The underlying mechanism of the effect of cilostazol in HFDinduced NAFLD mice.

hepatic steatosis by modulating hepatic STAMP2 expression through AMPK. Enhancing hepatic STAMP2 expression by cilostazol may be a potential therapeutic approach for the treatment of NAFLD.

\section{Authorship Contributions}

Participated in research design: Oh, Kim, Yoo.

Conducted experiments: Oh, Kim, M. H. Lee, Choi, Kwon.

Performed data analysis: Kim, Yoo.

Wrote or contributed to the writing of the manuscript: Suh, Choi, Nam, S. Y. Lee, Yoo.

\section{References}

Abedini A and Shoelson SE (2007) Inflammation and obesity: STAMPing out insulin resistance? Immunol Cell Biol 85:399-400.

Anstee QM and Goldin RD (2006) Mouse models in non-alcoholic fatty liver disease and steatohepatitis research. Int $J$ Exp Pathol 87:1-16.

Aoki C, Hattori Y, Tomizawa A, Jojima T, and Kasai K (2010) Anti-inflammatory role of cilostazol in vascular smooth muscle cells in vitro and in vivo. $J$ Atheroscler Thromb 17:503-509.

Arner P, Stenson BM, Dungner E, Näslund E, Hoffstedt J, Ryden M, and Dahlman I (2008) Expression of six transmembrane protein of prostate 2 in human adipose tissue associates with adiposity and insulin resistance. J Clin Endocrinol Metab 93:2249-2254

Beebe HG, Dawson DL, Cutler BS, Herd JA, Strandness DE Jr, Bortey EB, and Forbes WP (1999) A new pharmacological treatment for intermittent claudication: results of a randomized, multicenter trial. Arch Intern Med 159:2041-2050.

Bugianesi E, Leone N, Vanni E, Marchesini G, Brunello F, Carucci P, Musso A, De Paolis P, Capussotti L, Salizzoni M, et al. (2002) Expanding the natural history of nonalcoholic steatohepatitis: from cryptogenic cirrhosis to hepatocellular carcinoma. Gastroenterology 123:134-140.

Cohen JC, Horton JD, and Hobbs HH (2011) Human fatty liver disease: old questions and new insights. Science 332:1519-1523. 
Cusi K (2016) Treatment of patients with type 2 diabetes and non-alcoholic fatty liver disease: current approaches and future directions. Diabetologia 59:1112-1120.

Day CP and James OF (1998) Steatohepatitis: a tale of two "hits"? Gastroenterology 114:842-845.

Day EA, Ford RJ, and Steinberg GR (2017) AMPK as a therapeutic target for treating metabolic diseases. Trends Endocrinol Metab 28:545-560.

Dinicolantonio JJ, Lavie CJ, Fares H, Menezes AR, O'Keefe JH, Bangalore S, and Messerli FH (2013) Meta-analysis of cilostazol versus aspirin for the secondary prevention of stroke. Am J Cardiol 112:1230-1234

Elam MB, Heckman J, Crouse JR, Hunninghake DB, Herd JA, Davidson M, Gordon IL, Bortey EB, and Forbes WP (1998) Effect of the novel antiplatelet agent cilostazol on plasma lipoproteins in patients with intermittent claudication. Arterioscler Thromb Vasc Biol 18:1942-1947.

Fujita K, Nozaki Y, Wada K, Yoneda M, Endo H, Takahashi H, Iwasaki T, Inamori M, Abe Y, Kobayashi N, et al. (2008) Effectiveness of antiplatelet drugs against experimental non-alcoholic fatty liver disease. Gut 57:1583-1591.

Hubert RS, Vivanco I, Chen E, Rastegar S, Leong K, Mitchell SC, Madraswala R, Zhou Y, Kuo J, Raitano AB, et al. (1999) STEAP: a prostate-specific cell-surface antigen highly expressed in human prostate tumors. Proc Natl Acad Sci USA 96 $14523-14528$.

Ikewaki K, Mochizuki K, Iwasaki M, Nishide R, Mochizuki S, and Tada N (2002) Cilostazol, a potent phosphodiesterase type III inhibitor, selectively increases antiatherogenic high-density lipoprotein subclass LpA-I and improves postprandial lipemia in patients with type 2 diabetes mellitus. Metabolism 51: 1348-1354.

Jeon BH, Lee YH, Yun MR, Kim SH, Lee BW, Kang ES, Lee HC, and Cha BS (2015) Increased expression of ATP-binding cassette transporter A1 (ABCA1) as a possible mechanism for the protective effect of cilostazol against hepatic steatosis. Metabolism 64:1444-1453.

Jung YA, Kim HK, Bae KH, Seo HY, Kim HS, Jang BK, Jung GS, Lee IK, Kim MK, and Park KG (2014) Cilostazol inhibits insulin-stimulated expression of sterol regulatory binding protein-1c via inhibition of LXR and Sp1. Exp Mol Med 46:e73.

Kanuri G and Bergheim I (2013) In vitro and in vivo models of non-alcoholic fatty liver disease (NAFLD). Int $J$ Mol Sci 14:11963-11980.

Kim HJ, Moon JH, Kim HM, Yun MR, Jeon BH, Lee B, Kang ES, Lee HC, and Cha BS (2014) The hypolipidemic effect of cilostazol can be mediated by regulation of hepatic low-density lipoprotein receptor-related protein 1 (LRP1) expression. Metabolism 63:112-119.

Kim HY, Cho HK, Yoo SK, and Cheong JH (2012) Hepatic STAMP2 decreases hepatitis B virus X protein-associated metabolic deregulation. Exp Mol Med 44: 622-632.

Kim HY, Park SY, Lee MH, Rho JH, Oh YJ, Jung HU, Yoo SH, Jeong NY, Lee HJ, Suh S, et al. (2015) Hepatic STAMP2 alleviates high fat diet-induced hepatic steatosis and insulin resistance. J Hepatol 63:477-485.

Kimura Y, Tani T, Kanbe T, and Watanabe K (1985) Effect of cilostazol on platelet aggregation and experimental thrombosis. Arzneimittelforschung 35 (7A):1144-1149.

Kohjima M, Higuchi N, Kato M, Kotoh K, Yoshimoto T, Fujino T, Yada M, Yada R, Harada N, Enjoji M, et al. (2008) SREBP-1c, regulated by the insulin and AMPK signaling pathways, plays a role in nonalcoholic fatty liver disease. Int $\mathrm{J} \mathrm{Mol} \mathrm{Med}$ 21:507-511.

Korkmaz CG, Korkmaz KS, Kurys P, Elbi C, Wang L, Klokk TI, Hammarstrom C, Troen G, Svindland A, Hager GL, et al. (2005) Molecular cloning and characterization of STAMP2, an androgen-regulated six transmembrane protein that is overexpressed in prostate cancer. Oncogene 24:4934-4945.

Marchesini G, Bugianesi E, Forlani G, Cerrelli F, Lenzi M, Manini R, Natale S, Vanni E, Villanova N, Melchionda N, et al. (2003) Nonalcoholic fatty liver, steatohepatitis, and the metabolic syndrome. Hepatology 37:917-923.

Okuda Y, Kimura Y, and Yamashita K (1993) Cilostazol. Cardiovasc Drug Rev 11: 451-465.
Osborn O and Olefsky JM (2012) The cellular and signaling networks linking the immune system and metabolism in disease. Nat Med 18:363-374.

Ota H, Eto M, Kano MR, Ogawa S, Iijima K, Akishita M, and Ouchi Y (2008) Cilostazol inhibits oxidative stress-induced premature senescence via upregulation of Sirt1 in human endothelial cells. Arterioscler Thromb Vasc Biol 28:1634-1639.

Park SY, Lee JH, Kim KY, Kim EK, Yun SJ, Kim CD, Lee WS, and Hong KW (2008) Cilostazol increases 3T3-L1 preadipocyte differentiation with improved glucose uptake associated with activation of peroxisome proliferator-activated receptor- $\gamma$ transcription. Atherosclerosis 201:258-265.

Park SY, Shin HK, Lee JH, Kim CD, Lee WS, Rhim BY, and Hong KW (2009) Cilostazol ameliorates metabolic abnormalities with suppression of proinflammatory markers in a db/db mouse model of type 2 diabetes via activation of peroxisome proliferator-activated receptor gamma transcription. J Pharmacol Exp Ther 329:571-579.

Ramadoss P, Chiappini F, Bilban M, and Hollenberg AN (2010) Regulation of hepatic six transmembrane epithelial antigen of prostate 4 (STEAP4) expression by STAT3 and CCAAT/enhancer-binding protein alpha. J Biol Chem 285:16453-16466.

Rotman Y and Sanyal AJ (2017) Current and upcoming pharmacotherapy for nonalcoholic fatty liver disease. Gut 66:180-190.

Suzuki K, Uchida K, Nakanishi N, and Hattori Y (2008) Cilostazol activates AMP activated protein kinase and restores endothelial function in diabetes. $\mathrm{Am} J$ Hypertens 21:451-457.

Tanaka N, Aoyama T, Kimura S, and Gonzalez FJ (2017) Targeting nuclear receptors for the treatment of fatty liver disease. Pharmacol Ther 179:142-157.

Tani T, Uehara K, Sudo T, Marukawa K, Yasuda Y, and Kimura Y (2000) Cilostazol, a selective type III phosphodiesterase inhibitor, decreases triglyceride and increases HDL cholesterol levels by increasing lipoprotein lipase activity in rats. Atherosclerosis 152:299-305.

Targher G (2007) Non-alcoholic fatty liver disease, the metabolic syndrome and the risk of cardiovascular disease: the plot thickens. Diabet Med 24:1-6.

ten Freyhaus H, Calay ES, Yalcin A, Vallerie SN, Yang L, Calay ZZ, Saatcioglu F, and Hotamisligil GS (2012) Stamp2 controls macrophage inflammation through nicotinamide adenine dinucleotide phosphate homeostasis and protects against atherosclerosis. Cell Metab 16:81-89.

Wada T, Onogi Y, Kimura Y, Nakano T, Fusanobori H, Ishii Y, Sasahara M, Tsuneki $\mathrm{H}$, and Sasaoka T (2013) Cilostazol ameliorates systemic insulin resistance in diabetic $\mathrm{db} / \mathrm{db}$ mice by suppressing chronic inflammation in adipose tissue via modulation of both adipocyte and macrophage functions. Eur $J$ Pharmacol 707: 120-129.

Waki H and Tontonoz P (2007) STAMPing out Inflammation. Cell 129:451-452.

Wang SB, Lei T, Zhou LL, Zheng HL, Zeng CP, Liu N, Yang ZQ, and Chen XD (2013)

Functional analysis and transcriptional regulation of porcine six transmembrane epithelial antigen of prostate 4 (STEAP4) gene and its novel variant in hepatocytes. Int J Biochem Cell Biol 45:612-620.

Wellen KE, Fucho R, Gregor MF, Furuhashi M, Morgan C, Lindstad T, Vaillancourt E, Gorgun CZ, Saatcioglu F, and Hotamisligil GS (2007) Coordinated regulation of nutrient and inflammatory responses by STAMP2 is essential for metabolic homeostasis. Cell 129:537-548.

Wong VW, Chitturi S, Wong GL, Yu J, Chan HL, and Farrell GC (2016) Pathogenesis and novel treatment options for non-alcoholic steatohepatitis. Lancet Gastroenterol Hepatol 1:56-67.

Yoo SK, Cheong J, and Kim HY (2014) STAMPing into mitochondria. Int J Biol Sci 10:321-326.

Address correspondence to: Dr. Young Hyun Yoo, Department of Anatomy and Cell Biology, Dong-A University College of Medicine, 32, Daesingongwonro, Seo-Gu, Busan 602-714, Republic of Korea. E-mail: yhyoo@dau.ac.kr 